\title{
Updated evidence for left main coronary artery disease: Practice versus the consensus
}

\author{
Zhe Zheng, MD, PhD, ${ }^{a, b}$ Heng Zhang, MD, PhD, ${ }^{\mathrm{a}, \mathrm{b}}$ and Bo Xu, MBBS ${ }^{\mathrm{a}, \mathrm{c}}$
}

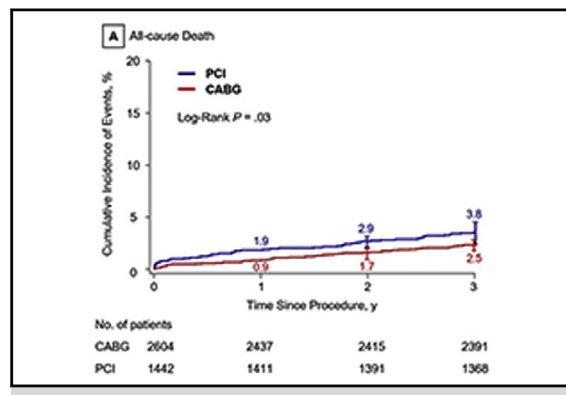

Unadjusted Kaplan-Meier curves of all-cause mortality according to revascularization strategy.

\section{Central Message}

The cumulative evidence on left main disease seems to achieve a consensus supporting the recommendation by the latest European Society of Cardiology guidelines on myocardial revascularization.

See Editorial Commentary page 314 .
Feature Editor's Note-The Expert Opinion that follows explores the pros and cons of percutaneous intervention (PCI) and coronary artery bypass grafting in patients with left main coronary disease, an important and controversial subset in the real world and in clinical trials. I recommend that surgeons keep an open mind about treating left main disease; although PCI is certainly not the gold standard, and likely is highly dependent on anatomic specifics, it is much safer and more effective than we used to think.

\section{Craig R. Smith, MD}

Coronary artery bypass grafting (CABG) has been recommended as the standard treatment for patients with unprotected left main disease (ULMD). However, with advances in interventional cardiology, percutaneous coronary intervention (PCI) is being increasingly used for ULMD, and outcomes of ULMD PCI have improved significantly over time. Importantly, based on the results from the Synergy Between Percutaneous Coronary Intervention with Taxus and Cardiac Surgery (SYNTAX) trial, the latest European Society of Cardiology (ESC) practice guidelines have updated the class of recommendation of PCI for patients with ULMD from class IIb to class I or IIa. ${ }^{1}$

The prespecified subgroup of the SYNTAX trial, by far the largest randomized trial in patients with ULMD reported to date, showed not only similar rates of death with CABG and PCI, but also comparable rates of major adverse cardiac events at 12 months, suggesting genuine clinical equipoise between the 2 approaches. ${ }^{2}$ Similarly, the 5-year results from the PRECOMBAT (Premier of Randomized Comparison of Bypass Surgery vs Angioplasty
Using Sirolimus-Eluting Stent in Patients with Left Main Coronary Artery Disease) trial suggest that PCI may be as effective as CABG for the treatment of ULMD.

In our recently published study, we compared outcomes between CABG surgery $(\mathrm{n}=2604)$ or PCI $(\mathrm{n}=1442)$ with drug-eluting stents in large consecutive Chinese cohorts during 2004 and 2010 (Figure 1). ${ }^{4}$ This is the largest real-world study published to date that has specifically evaluated and compared long-term outcomes in a cohort of consecutive patients with ULMD undergoing coronary revascularization. Moreover, unlike previously published randomized trials that were not powered primarily for comparing outcomes, our study was able to detect differences in endpoints, such as death, myocardial infarction, and stroke. Our findings show that in patients with ULMD, PCI is a reasonable alternative to CABG for patients with less complex disease (low to intermediate SYNTAX score; $\leq 32$ ), whereas CABG has a greater survival benefit compared with PCI (hazard ratio [HR], $1.71 ; 95 \%$ confidence interval $[\mathrm{CI}], 1.32-2.21 ; P<.001)$ in patients with a high SYNTAX score $(>32)$. Although previous studies have indicated consistent long-term outcomes with these 2 approaches, our present findings offer new evidence for a comparison between CABG and PCI, especially in consecutive populations of Chinese patients. In this regard, the cumulative evidence seems to 


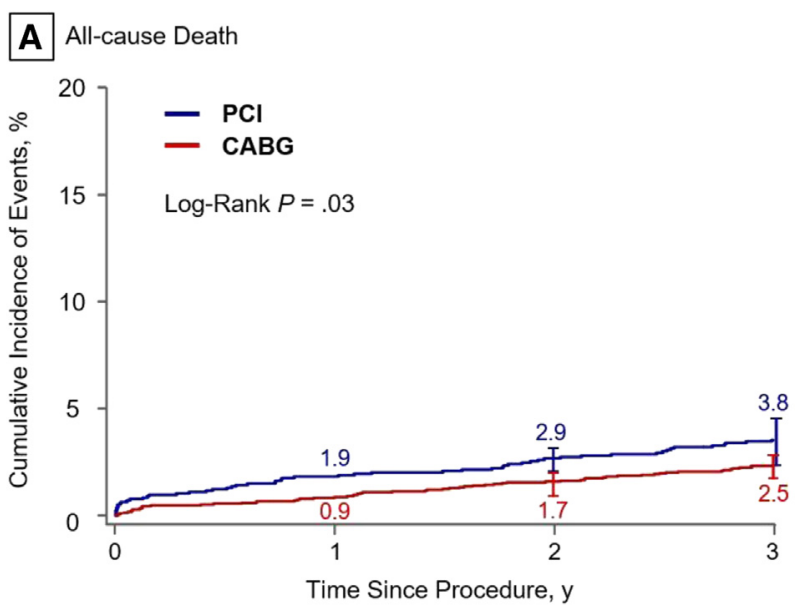

No. of patients

$\begin{array}{lllll}\text { CABG } & 2604 & 2437 & 2415 & 2391 \\ \text { PCI } & 1442 & 1411 & 1391 & 1368\end{array}$

FIGURE 1. Unadjusted Kaplan-Meier curves of all-cause mortality according to revascularization strategy. At 3 years after the procedure, the Kaplan-Meier estimate of all-cause mortality was 3.8\% in the PCI group, significantly higher than the $2.5 \%$ in the CABG group. The $P$ values were calculated using the log-rank test using all available follow-up data. $C A B G$, Coronary artery bypass grafting; $P C I$, percutaneous coronary intervention. Adapted with permission. ${ }^{4}$

achieve a consensus supporting the recommendations for myocardial revascularization published in the latest ESC guidelines.

Obviously, CABG has been documented to be more effective than PCI in relieving ischemia, achieving more complete revascularization, and thereby avoiding future clinical events. Thus, it should not be surprising that CABG remains the appropriate approach in patients with extremely advanced coronary artery disease, although the gap is narrowing. Nevertheless, the accumulated experience, coupled with improved technology and pharmacology, has led to the recent rapid evolution and broad adoption of PCI for treating ULMD. As such, the application of a clinical decision making system to evaluate CABG and PCI should be encouraged to best manage patients with ULMD. Although the SYNTAX score is useful for identifying patients at high risk undergoing PCI, outcomes after CABG are much less affected by anatomic complexity than those after PCI. Compared with the original, purely angiographic SYNTAX score, the recently developed SYNTAX score II has better long-term prognostic power in terms of mortality.

It should be noted that in numerous studies CABG has been associated with a lower risk of repeat revascularization but a higher risk of stroke compared with PCI, suggesting that the survival benefit after CABG must be balanced against the risk of stroke. The reported estimated incidence of early and delayed stroke is $2 \%$ to $9 \%$ after CABG and $0.15 \%$ to $0.44 \%$ after PCI. Patients undergoing CABG often have more complex conditions of coronary anatomy and perhaps greater predisposition to concomitant cerebrovascular disease; thus, establishing clinical directives for aggressive medical therapy in postrevascularization patients might result in decreased stroke risk, as well as lower rates of disability and healthcare utilization associated with stroke after CABG.

The optimum revascularization strategy for each individual patient should balance the risks and benefits associated with each procedure in conjunction with the patient's baseline risk profile and preferences. Moreover, the heart team approach, providing a balanced, multidisciplinary decision making process, is critical to minimize specialty bias and to achieve optimal patient care in the treatment of ULMD. The ongoing EXCEL (Evaluation of the XIENCE PRIME Everolimus-Eluting Stent System or XIENCE V EECSS vs Coronary Artery Bypass Surgery for Effectiveness of Left Main Revascularization) and NOBLE (Nordic-Baltic-British Left Main Revascularization) trials may confirm the safety and efficacy of CABG and PCI with later-generation drug-eluting stents in patients with ULMD.

\section{Conflict of Interest Statement}

Authors have nothing to disclose with regard to commercial support.

\section{References}

1. Windecker S, Kolh P, Alfonso F, Collet JP, Cremer J, Falk V, et al. 2014 ESC/EACTS guidelines on myocardial revascularization: the Task Force on Myocardial Revascularization of the European Society of Cardiology (ESC) and the European Association for Cardio-Thoracic Surgery (EACTS) developed with a special contribution of the European Association of Percutaneous Cardiovascular Interventions (EAPCI). Eur Heart J. 2014;35:2541-619.

2. Morice MC, Serruys PW, Kappetein AP, Feldman TE, Ståhle E, Colombo A, et al Five-year outcomes in patients with left main disease treated with either percutaneous coronary intervention or coronary artery bypass grafting in the Synergy Between Percutaneous Coronary Intervention with Taxus and Cardiac Surgery Trial. Circulation. 2014;129:2388-94.

3. Ahn JM, Roh JH, Kim YH, Park DW, Yun SC, Lee PH, et al. Randomized trial of stents versus bypass surgery for left main coronary artery disease: 5-year outcomes of the PRECOMBAT study. J Am Coll Cardiol. 2015;65:2198-206.

4. Zheng Z, Xu B, Zhang H, Guan C, Xian Y, Zhao Y, et al. Coronary artery bypass graft surgery and percutaneous coronary interventions in patients with unprotected left main coronary artery disease. J Am Coll Cardiol Intv. 2016;13:1102-11.

5. Campos CM, van Klaveren D, Farooq V, Simonton CA, Kappetein AP, Sabik JF III, et al; on behalf of the EXCEL Trial Investigators. Long-term forecasting and comparison of mortality in the Evaluation of the Xience Everolimus Eluting Stent vs. Coronary Artery Bypass Surgery for Effectiveness of Left Main Revascularization (EXCEL) trial: prospective validation of the SYNTAX Score II. Eur Heart J. 2015;36:1231-41. 